\title{
COMPARAÇÃO ENTRE DOIS PROCEDIMENTOS DE AMOSTRAGEM PARA INVENTÁRIO DA ARBORIZAÇÃO DO CENTRO URBANO DE GURUPI-TO
}

\author{
Dário Rabêlo ${ }^{1}$, André Ferreira dos Santos², Romualdo Júlio Cavalcante Wanderley${ }^{1}$, \\ Patrícia Aparecida de Souza ${ }^{3}$, Marcos Giongo ${ }^{4}$
}

\section{RESUMO}

Objetivou-se neste estudo comparar dois procedimentos de amostragem (o casual simples e o sistemático), avaliando a sua eficiência para o inventário das árvores do centro urbano de Gurupi-TO. Os dados foram coletados através do levantamento quantitativo total (censo) da arborização em 294 quadras com dimensões de 100 m x 100 m cada. Todas as árvores encontradas nas ruas e avenidas dentro de cada quadra com diâmetro a altura do peito (DAP) igual ou maior que $5 \mathrm{~cm}$ foram medidas. Para estimar o parâmetro populacional das árvores utilizou-se a variável "número de árvores por quadra". Do total das quadras ou unidades amostrais que compunham a área de estudo, foi obtida uma intensidade amostral de quase 13\% (38 quadras) para constituir a amostra dos procedimentos, tanto para 0 casual simples como para o sistemático. Os resultados da análise estatística mostram um erro de amostragem de $21,72 \%$ e $15,65 \%$ para os procedimentos casual simples e sistemático, respectivamente. Concluiu-se que o procedimento de amostragem sistemática é mais eficiente que o casual simples para fazer o inventário quali-quantitativo da arborização urbana da região central de Gurupi (TO).

Palavras-chave: Arborização urbana; Inventário arbóreo; Método de amostragem.

\footnotetext{
Recebido em 27.03.2014 e aceito em 26.05.2015

1 Engenheiros Florestais pela Universidade Federal do Tocantins (UFT), Campus de Gurupi, TO. Email: dario.rabelo@yahoo.com.br, julio_cavalcanti@hotmail.com.

2 Engenheiro Florestal, Dr., Professor Adjunto do Curso de Engenharia Florestal, Coordenador do Curso de Especialização em Educação Ambiental EaD - Universidade Federal do Tocantins (UFT), Campus de Gurupi, TO. Email: andrefs@mail.uft.edu.br.

3 Engenheira Florestal, Dra., Coordenadora do Curso de Engenharia Florestal da Universidade Federal do Tocantins - UFT, Campus de Gurupi,TO. Email: patriciaapsouza@mail.uft.edu.br.

4 Engenheiro Florestal, Dr., Professor Adjunto da Universidade Federal do Tocantins (UFT), Campus de Gurupi, TO. Email: giongo@mail.uft.edu.br.
} 


\section{COMPARISON BETWEEN TWO SAMPLING DESIGN FOR INVENTORY OF THE URBAN FOREST OF GURUPI (TO) CENTRAL AREA.}

\section{ABSTRACT}

The aim of this study was to compare two sampling designs (random sampling and systematic sampling) by the evaluation of their efficiency for the inventory of trees located in the urban center of Gurupi (TO). The data was acquired through the complete enumeration (census) of the urban forest in 294 blocks of $100 \mathrm{~m} \times 100 \mathrm{~m}$ dimensions each. All trees found in the streets and avenues within each block with diameter at breast height (DBH) equal to or greater than $5 \mathrm{~cm}$ were measured. For the estimation of the population parameter of the trees the variable "number of trees per block" was used. From the total blocks or sampling units that composed the study area, a sampling intensity of $13 \%$ (38 blocks) was obtained to form the samples for both random and systematic sampling designs. The statistical analysis results show a sampling error of $21.72 \%$ and $15.65 \%$ in the random and systematic sampling design, respectively. Thus, it was concluded that systematic sampling design is more efficient than random sampling to do the qualitative-quantitative inventory of the urban forest of the central area of Gurupi (TO).

Keywords: Urban forest; Inventory tree; Sampling design.

\section{INTRODUÇÃO}

O planejamento de qualquer recurso se baseia no conhecimento da situação atual, e para tanto, são utilizados inventários (MENEGHETTI, 2003).

O inventário é uma ferramenta fundamental para o estudo da arborização urbana, pois através dele pode-se conhecer o patrimônio arbóreo e identificar as necessidades de manejo. O método a ser utilizado depende basicamente das características do local e dos recursos disponíveis (SILVA et al., 2005).

A identificação da unidade amostral e a estrutura populacional constituem a primeira providência ao se planejar um inventário de arborização viária. Uma rua, um quarteirão, um grupo de quarteirões, uma quadra ou uma área de tamanho fixo pode constituir uma 
unidade amostral. A população é o número de unidades amostrais ou parcelas existentes na cidade ou bairro que se pretende avaliar. A amostra deverá ser retirada dessa população (COUTINHO, 1997).

O procedimento de amostragem trata da abordagem da população quanto ao conjunto das parcelas, isto é, como distribuirá as unidades amostrais ou parcelas dentro da área a ser inventariada.

De acordo com Soares et al. (2009), a amostragem aleatória é o método básico de seleção probabilística em que, na escolha de uma amostra composta de $n$ unidades de amostra, todas as possíveis combinações das $n$ unidades teriam as mesmas chances de ser selecionadas. Como não é um método perfeito, segundo Silva et al. (2007), apresenta algumas desvantagens como: dificuldade de localizar no campo, a posição das unidades de amostra dispersas na população; tempo improdutivo gasto no deslocamento entre as unidades de amostra e possibilidade de uma distribuição irregular das unidades.

A amostragem sistemática, por possibilitar uma melhor distribuição das unidades amostrais em relação à amostragem aleatória, e ao mesmo tempo produzir resultados mais precisos, vem sendo utilizada com maior frequência (COUTINHO, 1997).

Com base em estudos sobre a viabilidade de procedimentos de amostragem para o inventário de arborização urbana, é possível escolher o mais adequado para determinado local.

Dessa forma, o presente trabalho teve como objetivo comparar dois procedimentos de amostragem, o casual simples e o sistemático, isto é, avaliar qual deles é o mais eficiente para representar a população arbórea existente nas calçadas das ruas e avenidas da região central da cidade de Gurupi-TO.

\section{MATERIAIS E MÉTODOS}

\section{Área de estudo}

O levantamento foi realizado no município de Gurupi, localizado no extremo sul do Estado de Tocantins (Figura 1A), compreendido pelas coordenadas $11^{\circ} 37^{\prime} 30^{\prime \prime} \mathrm{S}$ e $49^{\circ} 07^{\prime}$ 30" W, tendo altitude média de 280 metros. O clima regional, segundo a classificação climática de Köppen (1948), é do tipo B1wA'a', úmido com moderada deficiência hídrica, a temperatura média anual é de $29,5^{\circ} \mathrm{C}$, com precipitação anual média de $1804 \mathrm{~mm}$, com verão chuvoso e inverno seco. 
Segundo o Instituto Brasileiro de Geografia e Estatística (IBGE), Gurupi está entre as três cidades mais populosas do Estado (Figura 1B) com população estimada em 2014 de 82.762 habitantes, com área de aproximadamente 1.836,081 $\mathrm{km}^{2}$ e com uma densidade populacional de um pouco mais que $40 \mathrm{hab} . / \mathrm{km}^{2}$.

Figura 1. Localização da área de estudo: A - município de Gurupi situado na região sul do Estado do Tocantins; $\mathrm{B}$ - perímetro da área de estudo dentro do município; C desenho representativo da área de estudo

Figure 1. Location of the study area: A - Gurupi municipality located in the southern region of the State of Tocantins, B - perimeter of the study area inside the municipality; $\mathrm{C}$ - representative drawing of the study area

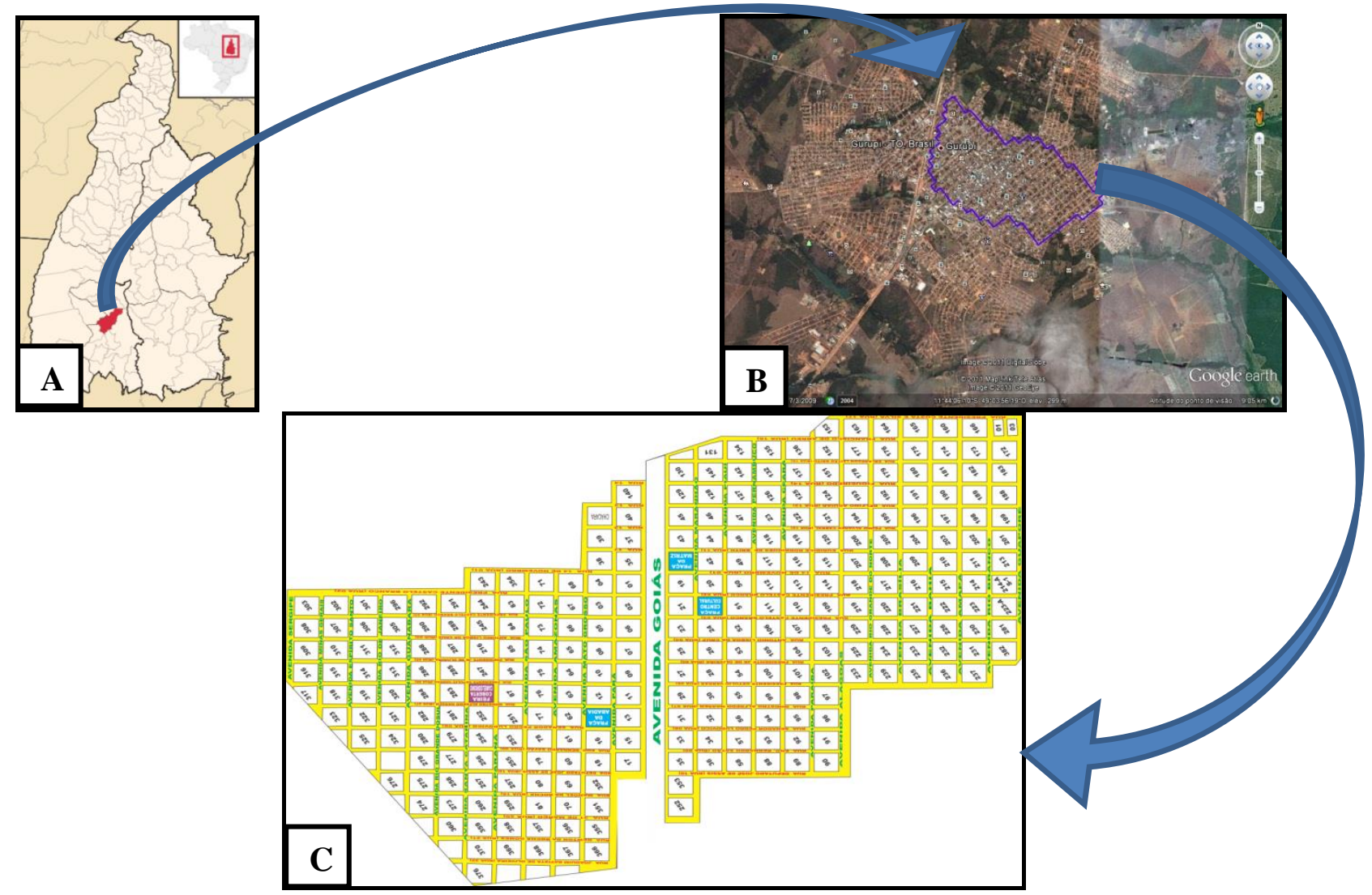

\section{Unidade amostral}

O estudo foi desenvolvido na região central de Gurupi, composta por aproximadamente 306 quadras com cerca de $10.000 \mathrm{~m}^{2}$ cada. Destas, foram inventariadas apenas 294 quadras, que eram mais semelhantes entre si ao formato de um quadrado.

O sistema de referência utilizado foi o mapa oficial do centro da cidade, contendo os números das quadras, nome das ruas, das avenidas e das praças, que serviu como modelo para ser desenhado pelo programa CorelDraw X5. Por meio dele foi possível a identificação 
das 294 quadras que compuseram a população. As quadras foram adotadas neste trabalho como unidades amostrais, a seguir definidas.

Cada unidade amostral (quadra) possui cerca de 100 metros de lado sendo que em seus lados oeste e leste passam avenidas e nos lados norte e sul as ruas (Figura 2).

Figura 2. Detalhe da área de estudo, mostrando os formatos das quadras $(A)$, e separadamente uma única unidade amostral com suas dimensões (B)

Figure 2. Detail of the study area, showing the shapes of blocks (A), and separately a single sampling unit with its dimensions $(B)$

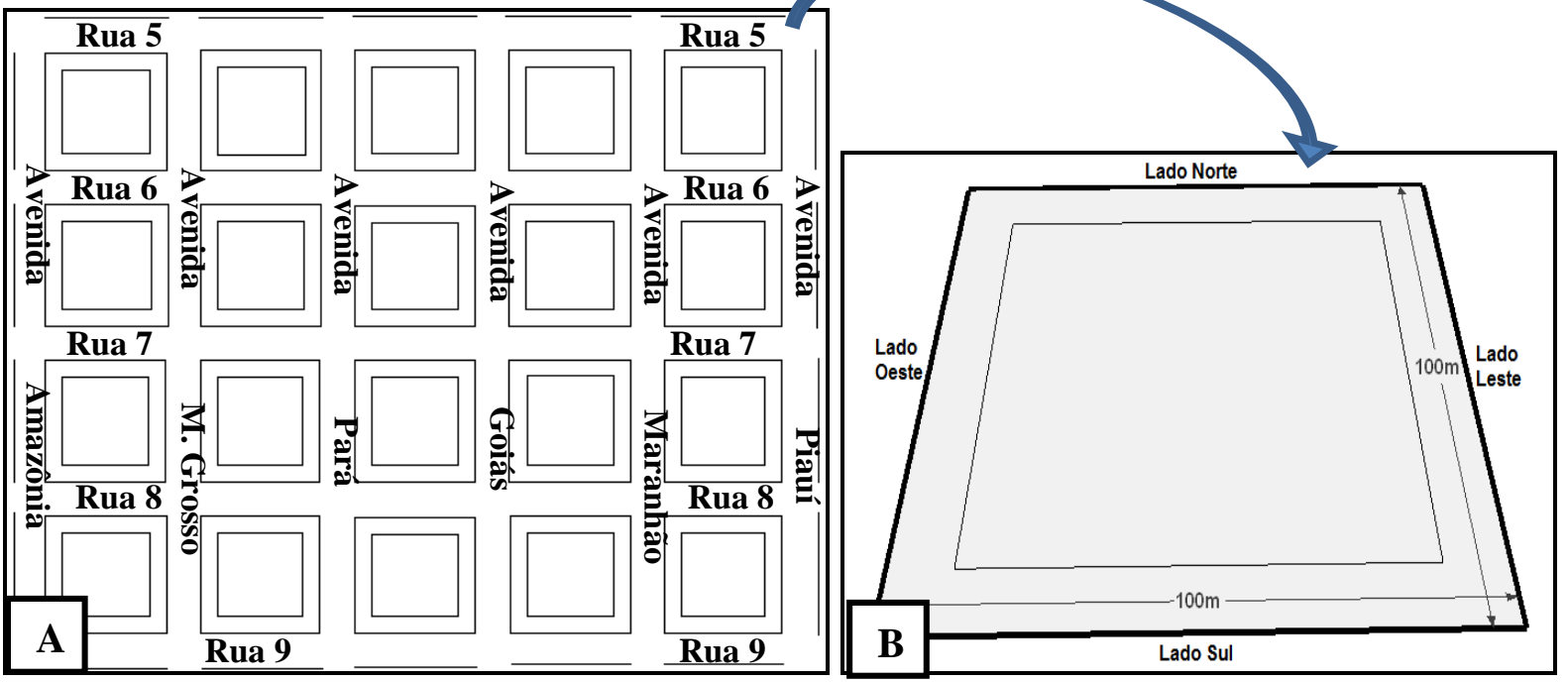

Do total das quadras encontradas na população, mais de $94 \%$ tinha o formato de um quadrado e o restante tinham os formatos semelhantes a um retângulo ou triângulo.

\section{Variável estudada}

A variável estudada neste inventário para estimativa dos parâmetros foi expressa em número de árvore por quadra e, também foi usado o número de árvores por quilômetro de calçada.

Pelo critério adotado, o total de árvores na calçada de uma quadra foi estimado, somando-se as árvores de seus quatro lados (norte, sul, leste e oeste), conforme exemplificado na Figura 3. 
Figura 3. Representação do número de árvores presentes em cada lado das calçadas de uma quadra

Figure 3. Representaion of the number of tree present on each sidewalk of a court

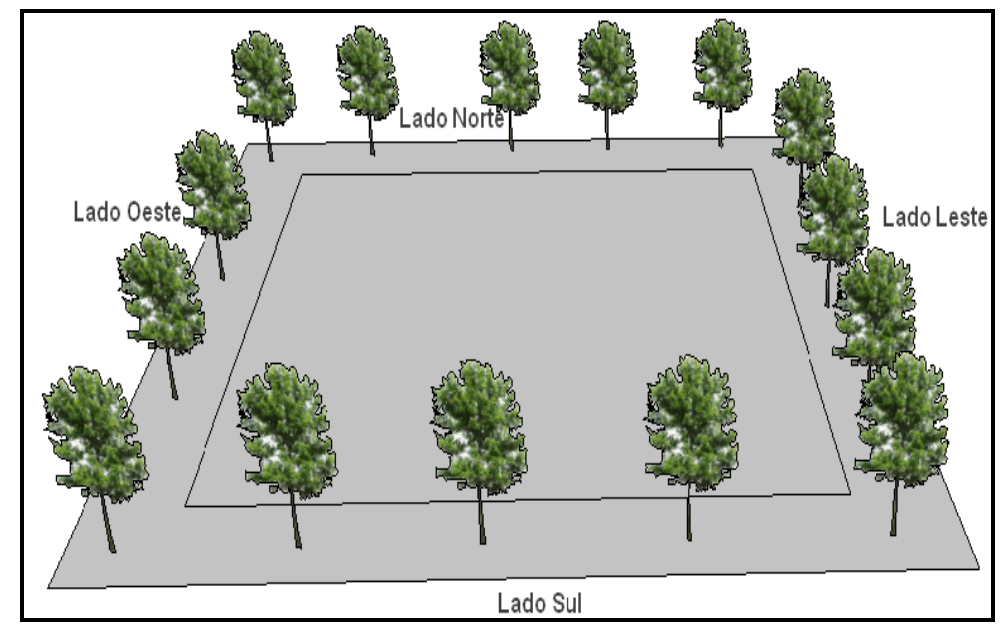

Para estimar o quilômetro de calçadas na área total, foi multiplicado o perímetro de uma quadra pela quantidade de unidades amostrais encontradas na população.

Em uma arborização urbana nas calçadas das quadras, os números de árvores usualmente apresentam alguma variação. Algumas quadras o número de árvores são maiores que a sua média aritmética, outras são menores e outras têm valores bem próximos da média. Evidentemente, o conhecimento sobre a dispersão dos números de árvores nessas áreas é importante. A média de dispersão mais comumente empregada para expressar essa dispersão dos dados, em relação à média, é a variância. Uma grande variância indica maior dispersão; uma variância pequena significa pouca dispersão. Dessa forma, para cada procedimento, a análise estatística foi baseada no formulário de Soares et al. (2009), aonde determinou-se a média; a variância; o desvio-padrão; o fator de correção; o erro médio-padrão; o erro de amostragem, tanto o absoluto quanto o relativo; o intervalo de confiança, tanto da média como da área total.

A eficiência de cada procedimento foi analisada segundo a intensidade de amostragem ideal (n), para obter-se um erro amostral de $20 \%$ com $95 \%$ de probabilidade da média verdadeira e o número de árvores na população de estarem dentro dos intervalos de confiança.

\section{Coleta dos dados}

O levantamento dos dados foi de caráter quantitativo, usando o método de área fixa (PÉLLICO; BRENA, 1997). Utilizou-se para amostragem os procedimentos de amostragem 
sistemática (AS) e o Casual simples (ACS) para se obter as unidades amostrais. Nas quadras (unidades amostrais), foram avaliadas apenas as árvores com o diâmetro a altura do peito (DAP) maior ou igual $5 \mathrm{~cm}$, medido com fita métrica.

De cada quadra selecionada anotou-se o total de árvores existente nas calçadas e o comprimento da mesma. Essas variáveis foram coletadas durante os meses de setembro, outubro e novembro do ano de 2013. No total de árvores foram incluídos todos os indivíduos arbóreos, arbustivos e herbáceos encontrados nessas áreas. Também foram inseridos no total de árvores os exemplares mortos.

\section{Inventário quantitativo total}

A realização do inventário quantitativo total objetivou fornecer os parâmetros populacionais usados como base para avaliação da eficiência entre os dois procedimentos de amostragem, sendo que os dados levantados foram digitados e processados em planilhas eletrônicas.

\section{Inventário quantitativo por amostragem}

O inventário quantitativo por amostragem foi utilizado para estabelecer comparações quanto à eficiência dos procedimentos de amostragem casual simples e amostragem sistemática em representar arborização da área de estudo.

Os dois procedimentos foram realizados observando-se o mapa da Figura 1, onde estão todas as quadras da região central da cidade. A intensidade amostral, isto é, a quantidade de unidades amostrais obtidas na área de estudo, foi de acordo com o total adquirido pela distribuição do procedimento sistemático que englobou toda a população.

\section{Amostragem sistemática e casual simples}

O procedimento de amostragem sistemática consistiu-se na seleção de unidades de amostra dentro da área total a partir de um esquema rígido e preestabelecido de sistematização, com os propósitos de cobrir toda a população.

No procedimento sistemático, a primeira amostra foi selecionada de maneira aleatória. A partir dela foi determinado uma distância na seleção de mais quadras, onde, na direção das avenidas, pré-estabeleceu uma distância de aproximadamente $100 \mathrm{~m}$ entre as 
unidades amostrais. Na direção das ruas, a distância entre as unidades amostrais foi maior, pouco mais que $300 \mathrm{~m}$, como está definida a seguir na Figura 4.

Figura 4. Trecho da população com algumas quadras selecionadas sistematicamente Figure 4. Portions of the population with some systematically selected blocks

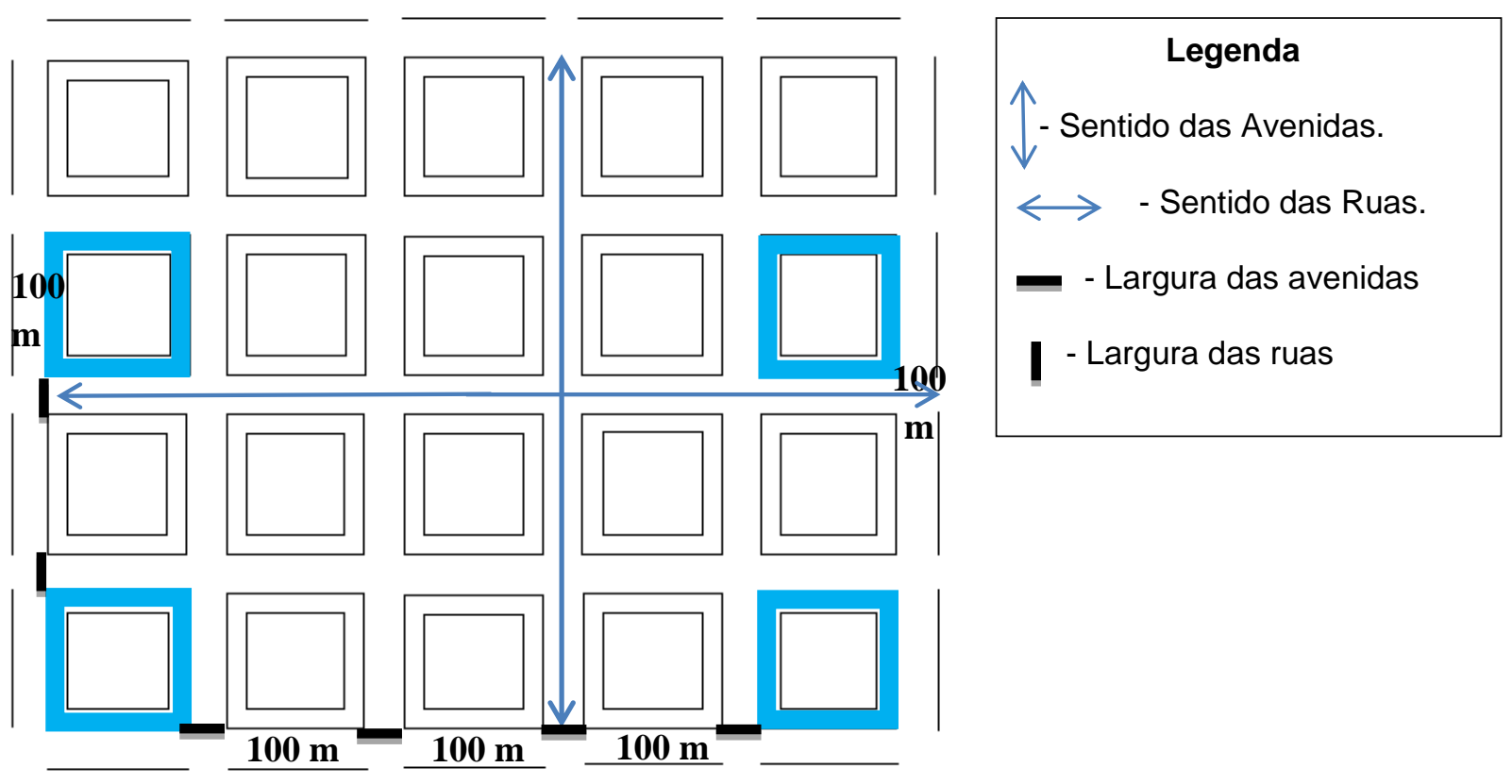

Com este procedimento, após cobrir toda a população, foi obtido uma intensidade amostral de quase $13 \%$, isto é, o equivalente a 38 quadras, usadas como unidades amostrais.

$\mathrm{Na}$ amostragem casual simples foi usada a mesma intensidade do procedimento anterior. Todas as 38 quadras foram selecionadas ao acaso dentro da população total, até atingir a fração amostral de aproximadamente 13\% na área de estudo.

\section{RESULTADOS E DISCUSSÃO}

\section{Inventário quantitativo total}

O censo feito para possibilitar comparações entre os dois procedimentos, indicou a presença de 3.601 indivíduos (arbóreo, arbustivo, palmeira) tanto vivos quanto mortos, nas 294 quadras da região central de Gurupi, com uma média de um pouco mais que 12 indivíduos por quadra. Apenas seis quadras apresentaram um pouco mais de $5 \%$ da arborização das 294 quadras amostrais, isto é, apenas seis quadras, foram encontradas 183 
plantas das 3.601 levantadas. As três quadras mais arborizadas foram: 86, 76 e 66, as quais foram vistos 32,31 e 30 , respectivamente, indivíduos.

O centro da cidade possui um percurso total de 117,6 quilômetros de calçadas presente nas margens das ruas e avenidas, com aproximadamente 31 indivíduos por quilômetro de calçada. Logo, esses indivíduos localizavam-se em maior proporção em dois lados da quadra, lado oeste (poente) e leste (nascente). Todavia, a explicação para isso, era porque essas áreas das calçadas não apresentavam muito a existência tanto da rede telefônica como também da rede de energia elétrica e, além disso, as calçadas eram mais largas, devido o fato de estarem presente nas margens das avenidas.

\section{Inventário por amostragem}

Com o procedimento de amostragem sistemática foram encontradas 436 exemplares (plantas vivas e mortas) presentes em um total de 15,2 quilômetros de calçadas. Obteve-se uma estimativa de quase 29 árvores por quilômetro de calçada, com uma média de aproximadamente 11 indivíduos por quadra.

$\mathrm{Na}$ amostragem casual simples, considerando a mesma intensidade amostral do procedimento anterior, foram encontradas nas calçadas das quadras sorteadas 438 plantas, tanto vivas quanto mortas, presentes na mesma distância do procedimento sistemático. Obteve-se a mesma estimativa de árvores por quilômetro de calçada do procedimento sistemático, todavia, com uma média de aproximadamente 12 indivíduos por quadra.

As estimativas dos parâmetros obtidos por ambos os procedimentos amostrais, as quais serviram de base para a discussão, estão na tabela 1.

Nota-se na tabela acima que a estimativa do erro-padrão da média apresentou menor valor absoluto para o processo de amostragem sistemática (0,88 árv./quadra) indicando maior precisão estatística em relação ao processo de amostragem casual simples (1,23 árv./quadra).

Esses resultados foram diferentes dos encontrados por Silva et al. (2005) na cidade de Belo Horizonte MG, onde os autores concluíram que o procedimento de amostragem casual simples foi mais preciso que o sistemático para aquela cidade.

Meneghetti (2003), em um estudo de dois métodos de amostragem para inventário da arborização de ruas dos bairros da orla marítima do município de Santos, SP, também indicou o uso da amostragem sistemática simples, uma vez que apresentou maior ganho em precisão. 
Tabela 1. Estimativas dos parâmetros obtidos pelos dois procedimentos, com os respectivos erros de amostragem e intervalos de confiança

Table 1. Estimation parameters obtained by the two designs, showing their sampling errors and confidence intervals

\begin{tabular}{c|c|c}
\hline Variável & Amostragem Sistemática & $\begin{array}{c}\text { Amostragem Casual } \\
\text { Simples }\end{array}$ \\
\hline$S^{2}(1)$ & 34,4722 & 65,7155 \\
$S(1)$ & 5,8713 & 8,1065 \\
$f$ & 0,8707 & 0,8707 \\
$S_{\bar{X}}(1)$ & 0,8887 & 1,2390 \\
$E(1)$ & 1,7962 & 2,5041 \\
$E \%$ & 15,6549 & 21,7252 \\
IC (Média) (1) & $11,47 \pm 1,7962$ & $11,52 \pm 2,50$ \\
IC (Total) & $2845,17-3901,34$ & $2652-4124,94$ \\
\hline
\end{tabular}

(1) Número de árvores por quadra.

Entretanto, a explicação para isso, pode ser embasada nas características da área em estudo, principalmente da variabilidade das unidades de amostra dentro da população, ou seja, as diferenças entre os números totais de árvores entre as unidades amostrais.

Portanto, de acordo com os erros de amostragem entre os dois procedimentos em questão, observou-se que apenas o procedimento de amostragem sistemática foi eficiente, pois ficou abaixo do erro pré-estabelecido. Além disso, o menor intervalo apresentado pelo intervalo de confiança obtido, tanto pela média de árvores por quadra quanto o número de indivíduos na população, vem reforçar o indicativo de sua maior eficiência em relação ao procedimento de amostragem casual simples.

\section{CONCLUSÕES}

Com base nos resultados pode-se dizer que há diferença quanto à eficiência dos procedimentos de amostragem casual simples e sistemática em representar a população arbórea que compõe a arborização urbana do centro de Gurupi-TO.

Portanto, observou-se que entre os dois procedimentos de amostragem, apenas 0 procedimento de amostragem sistemática foi eficiente com esta intensidade amostral (13\%), pois ficou abaixo do erro pré-estabelecido. 


\section{REFERÊNCIAS}

COUTINHO, L, C. Métodos de amostragem para avaliação da arborização viária. Mestrando em ciências ambientais e florestais. Universidade Federal Rural do Rio de Janeiro - UFRRJ. Rio de Janeiro. 1997.

IBGE. In: População estimada: Gurupi-TO. Disponível em: <http://cidades.ibge.gov.br/xtras/ perfil.php?lang=\&codmun=170950>. Acesso em: 13 out 2013.

KÖPPEN, W. 1948. Climatologia. México: Fundo de Cultura Econômica. 479p.

MELO, R. R.; FILHO, J. A. L.; JúNIOR, F. R. Diagnóstico qualitativo e quantitativo da arborização urbana no bairro Bivar Olinto, Patos, Paraíba. Revista da Sociedade Brasileira de Arborização Urbana, Patos, PB, v. 2, n. 1, 2007.

MENEGHETTI, G.I.P. Estudo de dois métodos de amostragem para inventário da arborização de ruas dos bairros da orla marítima do município de Santos, SP. Dissertação (mestrado) - Escola Superior de Agricultura "Luiz de Queiroz", Universidade de São Paulo, para obtenção do Título de Mestre em Recursos Florestais, Piracicaba, 2003.

MICHI, S. M. P.; COUTO, H. T. Z. Estudo de dois métodos de amostragem de árvores de rua na cidade de Piracicaba - SP. Revista IPEF. ESALQ/USP, 1996.

PÉLLICO S. N.; BRENA D. A. Inventário Florestal. Universidade Federal do Paraná Universidade Federal de Santa Maria. Curitiba. 1997.

RACHID, C.; COUTO, H. T. Z. Estudo da eficiência de dois métodos de amostragem de árvores de rua na cidade de São Carlos - SP. Scientia Forestalis, São Carlos, SP, n. 56, p. 59-68, 1999.

SILVA, A. G.; GONÇALVES, W.; LEITE, H. G. Estudo comparativo entre os procedimentos de amostragem casual simples e amostragem sistemática em inventário de arborização urbana. Natureza e desenvolvimento, Viçosa, MG, v. 1, n. 1, p. 67-73, 2005.

SILVA, A. G.; GONÇALVES, W.; PAIVA, H. N. Avaliando a arborização urbana. Vicosa, MG; Editora aprenda fácil, 346p. 2007.

SOARES, C.P.B.; NETO, F.P.; SOUZA, A.L. Dendrometria e Inventário Florestal. Viçosa, MG; Editora UFV, 275 p. 2009. 